Revue des patrimoines

\title{
Le petit jardin de Jean-Louis de La Valette à Cadillac (Gironde)
}

\section{Catherine Duboÿ-Lahonde}

\section{(2) OpenEdition}

\section{Journals}

Édition électronique

URL : http://journals.openedition.org/insitu/1155

DOI : 10.4000/insitu. 1155

ISSN : 1630-7305

\section{Éditeur}

Ministère de la culture

Référence électronique

Catherine Duboÿ-Lahonde, «Le petit jardin de Jean-Louis de La Valette à Cadillac (Gironde) », In Situ [En ligne], 1 | 2001, mis en ligne le 24 avril 2012, consulté le 21 avril 2019. URL : http:// journals.openedition.org/insitu/1155; DOI : 10.4000/insitu.1155

Ce document a été généré automatiquement le 21 avril 2019

\section{(c) $($ ) $(3)$}

In Situ Revues des patrimoines est mis à disposition selon les termes de la licence Creative Commons Attribution - Pas d'Utilisation Commerciale - Pas de Modification 4.0 International. 


\title{
Le petit jardin de Jean-Louis de La Valette à Cadillac (Gironde)
}

\author{
Catherine Duboÿ-Lahonde
}

Figure 1

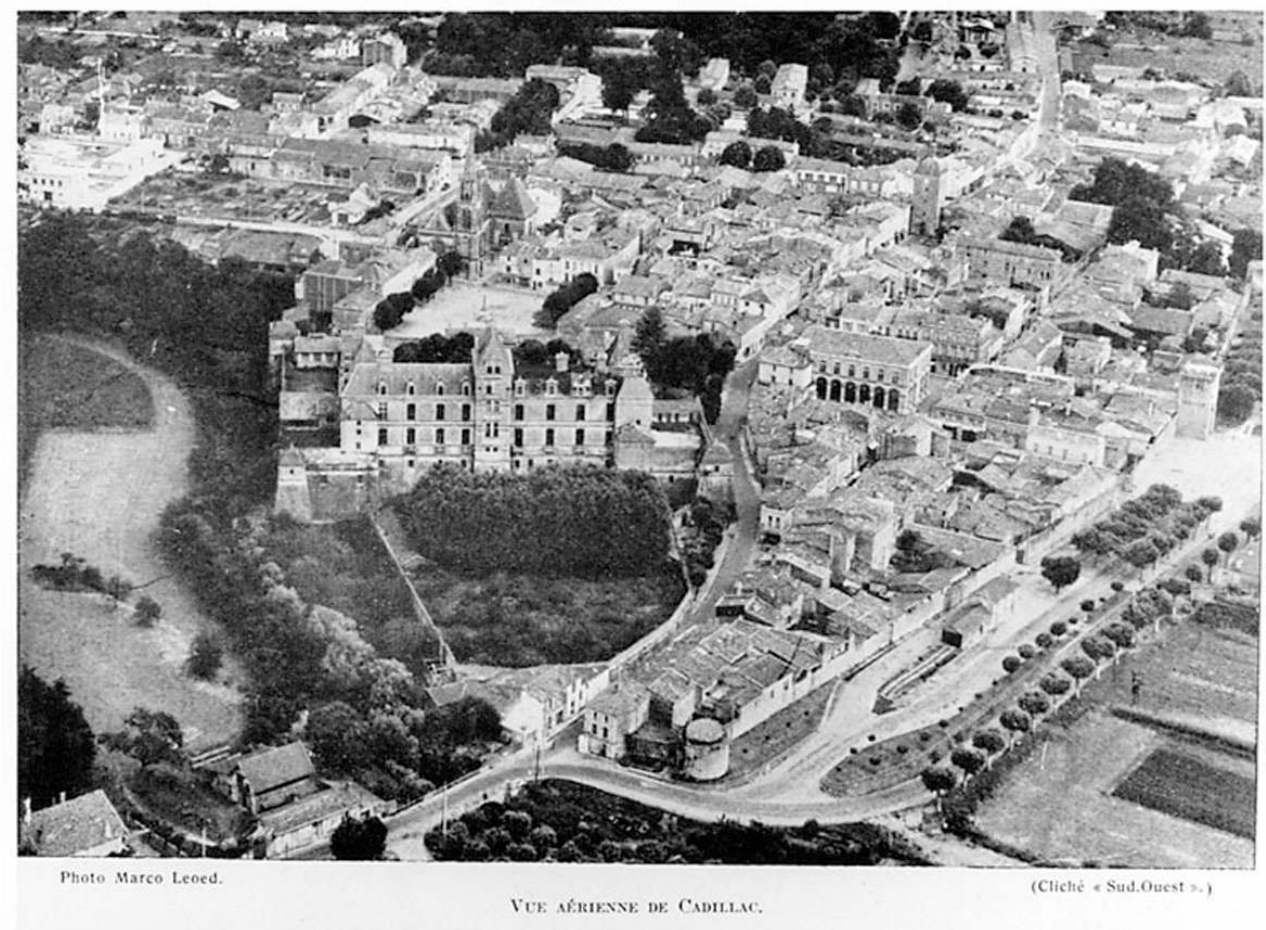

Situation du petit jardin. Vue aérienne du château en 1953

Phot. Marco Leoed (journal Sud-Ouest). Repro. Inv. M. Dubau (c) Inventaire général, ADAGP, 2001

Cadillac, 4 août 1599: pose de la première pierre du château commandé par le duc d'Epernon. En même temps que s'élève l'édifice, on aménage en contrebas de sa façade occidentale le petit jardin, ainsi nommé dans tous les comptes de travaux du château. Il 
ne reste évidemment rien de l'agencement de cet espace, mais des mentions dans les archives et les descriptions plus ou moins détaillées qu'en firent certains des voyageurs qui passèrent à Cadillac au XVII ${ }^{\mathrm{e}}$ siècle ${ }^{1}$ permettent d'en restituer une image approximative. Sous la direction du maître jardinier Jacques de Limoges ${ }^{2}$, qui est sur place avant janvier 1601, on le ferme de murs et on le nivèle à l'aide de la terre tirée du fossé qui longe la façade. Sa forme irrégulière, due à sa situation à un angle des fortifications de la ville, dont un des murs lui sert de clôture au nord ${ }^{3}$, le fait qu 'il soit clos et son accès depuis le logis par un pont au-dessus d'un profond fossé confèrent à ce jardin un caractère privé encore médiéval. Jean Le Laboureur note en 1659 que le " parterre ne répond pas à la majesté du bâtiment, tant parce qu'il faut descendre pour y aller, que pour être petit et enfermé ". Sa structure ne semble pas avoir été pensée en même temps que l'édifice, dont l'assise est encore celle d'une forteresse ; cependant, la présence dès les débuts du chantier d'un jardinier formé dans un milieu où l'on recherche l'harmonie entre l'édifice et son environnement indique une volonté d'utilisation cohérente de l'espace.

2 C'est lorsqu'on évoque le contenu du jardin que sa modernité se révèle. Le 6 janvier 1607, les comptes mentionnent le " jardin de fleurs », puis en 1612, Pierre Bergeron note qu'il " n'est que pour fleurs ". Dès sa conception, c'est donc un jardin au goût du jour ${ }^{4}$, un de ces jardins fleuristes qui furent tant prisés dans la première moitié du XVII siècle dans les milieux savants ou proches de la cour. On peut imaginer qu'à Cadillac, tout comme à Paris, fleurirent bulbes et oignons, notamment de ces tulipes de Hollande dont l'engouement fut alors si fort ${ }^{5}$; probablement plantées dans des compartiments entourés de palissades basses ou de bordures de thym ou d'hysope et divisés par des allées, elles se trouvaient certainement en compagnie d'autres fleurs tout aussi à la mode, anémones, renoncules, iris ou jacinthes, mais aussi de rosiers odorants et d'arbustes, peut-être même déjà d'orangers en caisse ${ }^{6}$. 
Figure 2

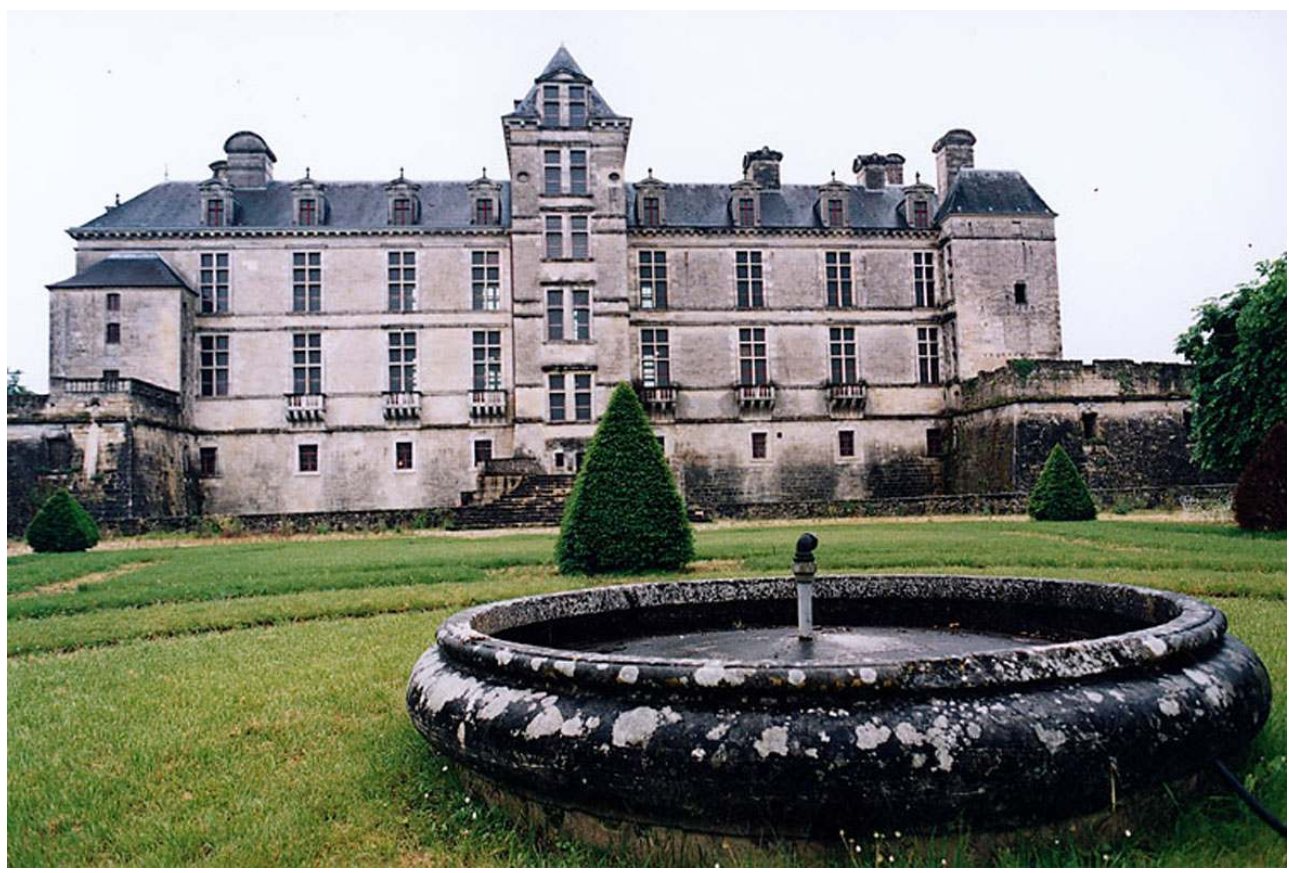

L'élévation occidentale du château, le jardin avec un des bassins de la fontaine installée en 1604. Etat actuel

Phot. Inv. M. Dubau. (c) Inventaire général, ADAGP, 2001

3 Au centre du jardin, déporté vers le nord par rapport à l'axe de la façade et du pont d'accès, le maître fontainier Jean Duferrier ${ }^{7}$, habitant Cadillac dès janvier 1600, installe en 1604 une fontaine jaillissante composée de deux bassins circulaires superposés, le plus grand en pierre, celui du dessus (d'environ 3 pieds de diamètre) en marbre ${ }^{8}$, monolithe "pour servir de bassin à un griffon »; un des deux bassins subsiste à son emplacement d'origine, l'autre se trouve dans une propriété privée. * 
Figure 3

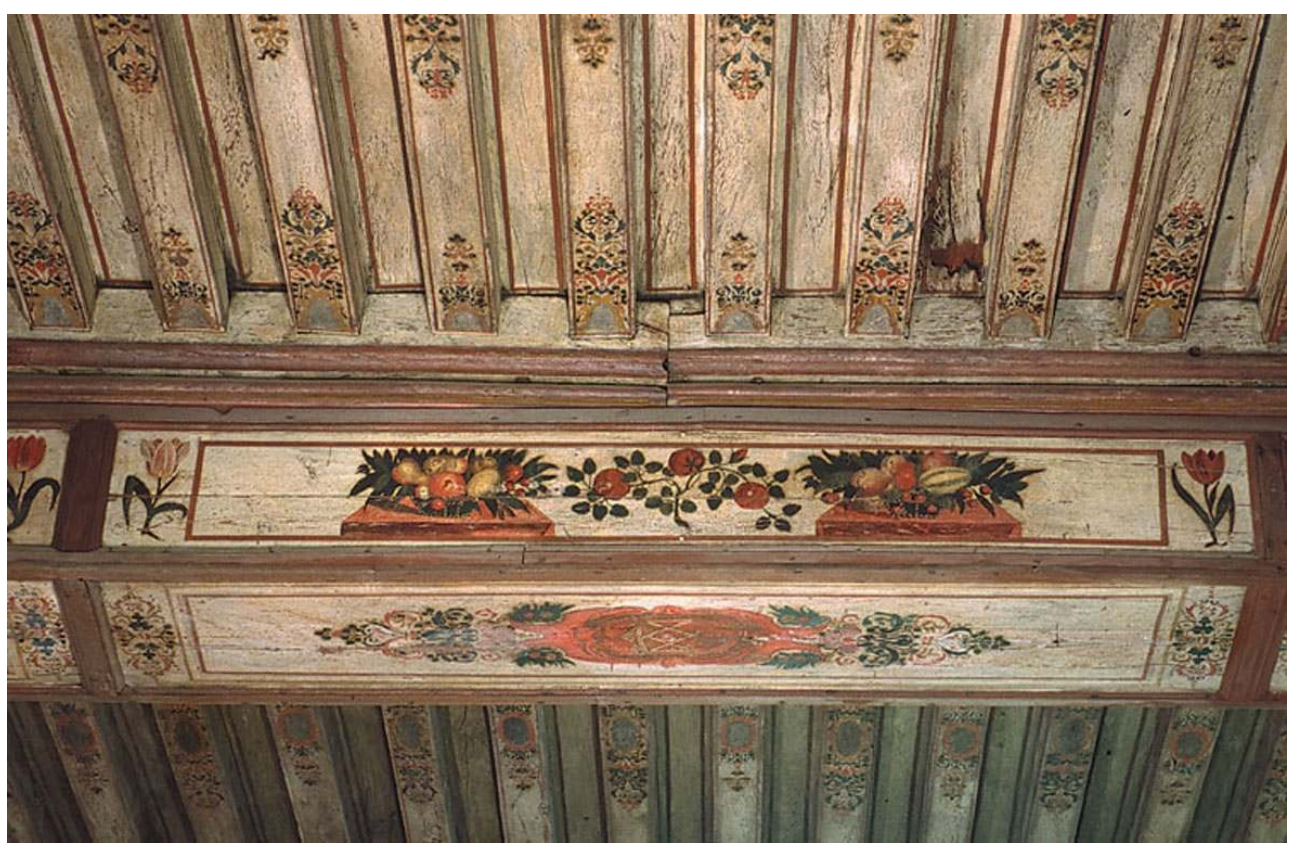

Antichambre de la duchesse. Décor peint vers 1614 par Girard Pageot

Phot. Inv. M. Dubau (c) Inventaire général, ADAGP, 2001

On ne trouve plus dans les comptes de mention du petit jardin jusqu'en 1632, date à laquelle le maçon Pierre Coutereau ${ }^{9} \mathrm{y}$ avait entrepris la maçonnerie de la "grotte et préau » (enclos) ; cette maçonnerie s'étant alors écroulée, elle ne sera reconstruite qu'en 1634, alors qu'est présent à Cadillac l'Angevin Jean Joullain de La Barre, «maître des grottes " du duc ${ }^{10}$. Jean Le Laboureur, en 1659, découvre " au bout de ce parterre...une salle d'armes ${ }^{11}$, et au milieu une fort belle grotte de coquillages avec des personnages de la fable ". Il paraît probable que Jean Joullain de La Barre fut ici rocailleur et l'auteur du programme décoratif de cette grotte où quelques nymphes et divinités aquatiques ne pouvaient manquer d'apparaître au visiteur. Il est assisté dans son travail par un certain Jacques Godin, lui aussi angevin et " maitre des grottes et fontaines " du duc, qui pourrait avoir conçu la partie hydraulique de l'ouvrage. La grotte est en effet agrémentée de jeux d'eau, suffisamment remarquables pour que Le Laboureur les décrive en détail : " de cette grotte il sort des jets d'eau sans nombre, comme aussi des environs, qui se voûtent en arc et autour desquels on peut se promener, mais il y a des tuyaux qui montent le long des ormes, qu'ils embrassent, dont on fait plusieurs malices en tirant les faussets pour mouiller ceux qui ne s'en défient pas ; la même malice se fait à l'entrée de la salle d'armes, à côté de laquelle est le regard pour faire jouer les eaux. A l'entrée de la grotte est un tuyau principal qui jette de l'eau en diverses figures, selon les moules qu'on y applique, et il y a une queue de tuyau courbe, dont on mouille de loin qui l'on veut ". On ignore l'emplacement de cette grotte dans le jardin; Le Laboureur semble la situer à son extrémité ouest, place où elle aurait fermé la perspective sur le paysage environnant, mais qui serait la plus commode pour la mise en œuvre des jeux d'eau, dont certains utilisaient le tronc d'ormes qui devaient exister auparavant. Un texte cependant suggère une autre situation pour la grotte. En effet, une lettre concernant la démolition partielle du château, réalisée vers 1758 , mentionne « la...muraille qui est du côté et sur le bord du fossé de ce qu'on appelle la grotte..., le pont qui communique du château à la dite 
grotte ", ce qui indiquerait un emplacement plus proche de la façade occidentale. Quoi qu'il en soit, en 1630, le duc songe, avec sa grotte de coquillages, à sacrifier à la mode en donnant à son petit jardin un air italien, certes plus modeste qu'à Wideville (Yvelines) qui lui est contemporain ${ }^{12}$, mais avec toutes les innovations apportées par les ingénieurs hydrauliciens italiens. Il perpétue en tout cas cette tradition des jeux d'eau à surprises qui, à l'instar de ceux du parc des ducs de Bourgogne à Hesdin au XV siècle, demeurent en vogue jusque vers le milieu du XVII ${ }^{e}$ siècle et correspondent à cet amour de la facétie et de l'enchantement si caractéristique de la sensibilité du temps. On ne saurait pourtant qualifier de facétieux le rude soldat que fut le duc d'Epernon ; il faut peut-être voir alors, dans la construction de cette grotte et de ses jeux d'eau, un acte ultime de grandeur et comme un dernier manifeste de la puissance de celui qui avait été un « demi-roi » ${ }^{13}$.

\section{NOTES}

1. Pierre Bergeron en 1612 et Jean Le Laboureur en 1659.

2. Il est fils de Louis de Limoges, « jardinier du roi », en réalité un des jardiniers du château royal de Montceaux-en-Brie.

3. Ce jardin occupe probablement une partie de la basse-cour du château médiéval que le duc d'Epernon fit démolir pour le remplacer par l'édifice actuel. Il appartenait à sa femme Marguerite de Foix-Candale.

4. Il est contemporain de celui de Jean Robin à Paris ; Marie de Médicis qui le fréquentait en fit dessiner les fleurs, ce qui aboutit en 1608 au recueil des 73 planches gravées du Jardin du Roy très chrétien Henri IV.

5. Les tulipes du jardin servirent peut-être de modèle au peintre Girard Pageot qui, vers 1614, en sema le plafond et les murs de l'antichambre de la duchesse.

6. En 1659, il y a plus de cent orangers à Cadillac.

7. Il est ingénieur et maître des réparations des bâtiments du roi de Navarre en 1585-1597.

8. Ce bassin, amené par voie fluviale depuis Thouars « en Condomois » (canton de Lavardac, Lotet-Garonne), est payé en octobre 1604.

9. Il est le fils de Louis Coutereau, maître-maçon du duc depuis les débuts du chantier.

10. Jean Joullain de La Barre demeure à Cadillac de 1630 à 1640. Il exécuta, lors de la venue de Louis XIII à Angers en 1614, deux figures sculptées et peintes placées à l'entrée du portail SaintAubin et représentant le fleuve Maine et la nymphe Daphné.

11. Aucun autre texte ne fait état de cette salle d'armes.

12. On peut imaginer que le « préau » prévu ait été ,comme à Wideville, un enclos pour un bassin.

13. Jean-Louis de La Valette, duc d'Epernon (1554-1642). Exilé à Loches en 1641, il y meurt à 88 ans le 13 janvier 1642. 
INDEX

Mots-clés : inventaire général, en ligne, journal, revue électronique, revue numérique, périodique, patrimoine, histoire de l'art, France, Aquitaine, Gironde, Cadillac, 17e siècle, La Valette Jean-Louis

Keywords : on line, electronic journal, ejournal, heritage, history of art, France, Aquitaine, Gironde, Cadillac, XVIIth century, La Valette Jean-Louis

\section{AUTEUR}

\section{CATHERINE DUBOŸ-LAHONDE}

Conservateur en chef, Service régional de l'Inventaire DRAC Aquitaine 54, rue Magendie 33074 Bordeaux cédex. catherine.duboy-lahonde@culture.gouv.fr 